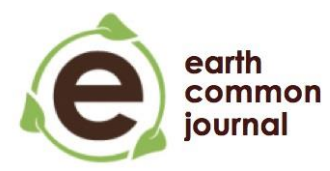

Earth Common Journal

Convergence

MacEwan University

Volume 5, Number 1, September 2015

\title{
Article
}

\section{Ecuador: Communicating to bridge the education gap in nutrition}

Brittany Pitruniak, Kathryn Adachi, Morgan Messelink

MacEwan University, Canada

\begin{abstract}
After conducting a study in May of 2014 on nutrition communication in Ecuador and how it affects consumer behaviour, researchers identified a gap between the level of education obtained by students and the knowledge of nutrition provided to them. This article uses that study to assess whether or not the communication of nutrition information can be understood with the level of nutrition education available through cultural and educational means. Researchers first gathered data using quantitative methods in a survey administered to students at the Universidad San Francisco de Quito. The data was supplemented through qualitative research in a questionnaire administered in a one-on-one interview format, followed by content analysis of food labels found in Ecuadorian grocery stores. Review of all of the data showed a number of problems with the level of education provided to students on the topic of nutrition and the communication of the information available to them. The inconsistency of food labeling in Ecuador also presented an issue. Researchers' recommendations for the sustainability of healthy living include a standardized curriculum regarding nutrition starting in primary school in order to influence a sustainable level of nutrition education, as well as a standardized and mandatory format for food labelling.
\end{abstract}




\section{Introduction}

In May of 2014, a research study was completed in Quito, Ecuador on how nutrition is communicated to consumers and their healthy eating behaviours in the Ecuadorian culture. Using a survey, and in-depth questionnaire, and content analysis of food labels, researchers were able to determine what the subjects considered a healthy diet, how this was communicated to consumers, and the extent to which the nutritional information communicated via food labels influenced students aged 18-25 in their eating behaviours.

An unexpected result of the study was the introduction of the question of whether or not the education available was sufficient enough to understand the communication presented on the food labels, as the convergence of education and communication appeared to have a substantial effect on the eating habits of the participants. While this was not one of the original research questions, the study allowed researchers to discover what was perceived as a major link between the nutrition education provided by the government and food manufactures, the level of understanding of the nutritional information communicated to consumers, and how these factors affected consumer behaviour.

\section{Significance of Study}

A healthy lifestyle is a current trending topic in Canadian culture. External influences, such as society and the media, pressure individuals to create and maintain a healthy lifestyle. A rising concern in Ecuador is obesity. A recent statistic shows that three out of 10 children in the country are overweight ("The traffic," 2014, para. 1). However, while obesity continues to be a growing problem, the issue is acknowledged by the Ecuadorian government and has prompted some changes to food labeling practices.

Ecuador is categorized under the group "mandatory" when it comes to statutory regulations on nutrition labelling, meaning they are obliged to "define which nutrients must be listed and on what basis (e.g. per 100g/ per serving)" (European Food Information Council, 2011, p. 4). In 2013, the Ministry of Health of Ecuador declared that packaged foods must carry mandatory traffic light labels ("The traffic," 2014, para. 1). The key objective of this traffic light labelling is to communicate nutrition information to consumers and help them make healthier food choices (Sacks, Rayner, \& Seinburn, 2009, p. 345). These labels are placed on food products to indicate fat, sugar, and salt content; "a 'red' light indicates a 'high' level of that nutrient, an 'amber' light indicates a 'medium' level, and a 'green' light indicates a 'low' level” (p. 345). However, researchers found no evidence that the traffic light labels were being implemented and the labels that 
were available were often inconsistent or incomplete. This is seen as a huge concern that could easily contribute to the misinterpretation or disregard of the information presented.

\section{Methodology}

The original study was conducted using a mixed method approach, beginning with a quantitative survey designed with only close-ended questions regarding demographics, instruction received, and consumer behaviour. However, only three of the survey questions will be examined for the purposes of this article. Out of the 71 survey participants, 10 agreed to participate in an in-depth, qualitative questionnaire administered in a one-on-one interview format; five of the 13 questions included in the questionnaire will be examined for the purposes of this article. All of the participants were between the ages of 18 and 25, and were students at Universidad San Francisco de Quito. Researchers concluded research by analyzing the labeling on over 60 food packages, including both back-of-pack (BOP; nutritional content) and front-of-pack (FOP; phrases such as 'fat free' or 'low in sugar') labels.

\section{Results}

The research conducted shows a startling trend in the relationship between the education provided, the communication available, and the subsequent understanding and consumer behaviour. For instance, $70 \%$ of the questionnaire participants answered 'no' when asked if they had ever received any formal instruction regarding nutrition and diet, leading researchers to believe that there is no structure in Ecuador's education system around teaching students about nutrition and the importance of healthy eating. Furthermore, $60 \%$ of these participants continued by stating that they learned what constitutes a healthy diet at home or from family members. This indicates that in traditional Ecuadorian culture, it is the responsibility of the family to educate their children on the importance of healthy eating, as well as the benefits of following a healthy diet. With the introduction of processed foods in the last two decades, there is a need to educate children outside of the family unit to ensure consistency between the information being taught by families, the Ecuadorian government, and product manufacturers.

Understanding the information available on food labels was indicated as a challenge for $47.9 \%$ of survey participants and $50 \%$ of the questionnaire participants. This shows an alarming problem, potentially with what information is on the label, the way it is presented on the label, and the nutrition education provided to consumers. Content analysis of the collected food labels showed an interesting trend of inconsistency in Ecuadorian nutrition information communication, particularly in the examination of BOP 
food labels. Of the 16 variables analyzed, research showed that only one of the categories (caloric content) was available on $100 \%$ of the labels gathered, with eight appearing on fewer than $80 \%$ and only four appearing on more than $95 \%$ of the collected labels.

Additionally, studies have shown that healthy eating in the Ecuadorian culture is associated with food preparation, rather than nutritional content. Traditional and homegrown foods are deemed "always healthy", as these are "hygienically prepared at home", and in contrast, "street or restaurant foods and food out-of-home in general were perceived as unhealthy because preparation methods were unknown" (Verstraeten, Van Royen, Ochoa-Avilés, Penafiel, Holdsworth, Donoso, 2014, p. 3). Because meals prepared and served in the home are considered automatically healthy to many Ecuadorians, it cannot be guaranteed that the actual food ingested is meeting all the nutritional requirements. A variety of products from all four food groups need to be consumed and a balance between these food groups achieved; two factors researchers cannot guarantee will be met by simply eating food prepared in the home. Because there is not a strong communication channel in Ecuador to educate the population on nutrition, there is a gap in the basic knowledge Ecuadorians have about nutrition and healthy living. Without this base knowledge, consumers are unable to make educated choices when purchasing groceries, as they are unable to fully understand the nutrition labels on food products.

The lack of education was also represented in a survey question asking how participants would describe the quality of their instruction regarding nutrition and diet, to which $76.1 \%$ of participants indicated 'average' to 'extremely poor' or 'none' on the Likert scale provided by the survey. Alzate, Dárdano, Olivares, and Álvarez (2012) conducted an evaluation of the training of nutritionists in eight South American countries, and found there are significant gaps in their curriculum model. It showed that less than half of the institutions provided instruction on "design and validation of nutrition education materials" (Alzate et al., 2012, p. S63), and that there is no clear standard of education in these professions. This indicates that nutritionists and dietitians in South America are often not properly trained in regards to communicating basic nutrition information such as daily intake and resource management to unique individuals. Nutrition education is a huge contributing factor in the decrease in obesity, and educators not being properly trained in nutrition, which can lead to further misunderstanding of healthy living.

Finally, the questionnaire asked participants if they thought the availability of nutrition information could be improved, to which $100 \%$ of interview participants answered 'yes'. This overwhelming statistic exemplifies the lack of nutrition base knowledge in Ecuador, and the people's desire to be better informed about nutrition. 
These questionnaire participants represent Ecuador's future, and their interest in nutrition could aid in the prevention of health risks and diseases. The unanimous response to needing to improve the availability of nutrition information indicates that Ecuadorians do care about their nutrition and want to learn more, but an internal struggle between cultural norms and a lack of education prevents many from moving forward toward a healthy future.

\section{Recommendations}

In reviewing all research data, there is a clear need for an increase in availability and clarity of the nutritional education provided to Ecuadorian citizens. Improvements in both of these areas would increase nutrition awareness among consumers, and as a result, begin to influence consumer behaviour. Mandatory nutrition classes offered in primary and secondary school could help to solve the issue, and some participants even suggested TV programming or campaigns to educate the general public. The key factor needed for success is that the solution be accessible and available to everyone. Education combined with improved communication, including a mandatory and standardized format for food labels, will help create a standard of healthy living that all Ecuadorian citizens can work to uphold. Stakeholders such as governments, international organizations, individual companies, and non-government organizations can be influential in making nutrition information more accessible to consumers through tactics such as educational programming, websites, advertising, and nutrition labelling systems (European Food Information Council, 2011, p. 6). This information will be useful to address health issues, such as defining healthy living and eating standards present in Ecuador, as well as working proactively to prevent future health risks.

*Authors:

Brittany Pitruniak: A recent graduate of MacEwan's Communication Studies program, Brittany continues her time at MacEwan as the President of the Students' Association of MacEwan University and a member of the MacEwan Board of Governors. Brittany is a passionate advocate. In the community, Brittany is the Vice Chair of Communications for Equal Voice Alberta Northern Chapter (EVAN). A lover of travel and change, you will find Brittany embracing both in all of her roles.

Kathryn Adachi: Kathryn is a third-year Communication Studies student majoring in Journalism at MacEwan University. A lifelong writer and artist, she has used her time at MacEwan to further her knowledge in all fields, including photography and graphic design. She has won a number of awards for her achievements in Journalism studies. 
Morgan Messelink: Morgan is a fourth year professional communications student at MacEwan University. Before submerging in the field of communications, I studied Nutrition and Food Science at the University of Alberta. My interest in both nutrition and communications were put to good use throughout the entirety of this article. I also explored this topic a bit while volunteering in Ghana so it was neat to see how the two experiences compared. Having this article published is a great accomplishment for Brittany, Kathryn, and myself.

\section{References}

Alzate, T., Dárdano, C., Olivares, S., \& Álvarez, C. (2012). Nutrition education of nutritionists: Worrying gaps in South America. Journal of Nutrition Education and Behavior, 44 (4), S63.

European Food Information Council. (2011). Global update on nutrition labeling [PDF]. Retrieved from The European Food Information Council website:http://www.eufic.org/upl/1/default/doc/GlobalUpdateExecSumV2Aug2 011 press.pdf

Sacks, G., Rayner, M., \& Swinburn, B. (2009). Impact of front-of-pack 'traffic-light' nutrition labeling on consumer food purchases in the UK. Health Promotion International, 24 (4), 344-352. Doi:10.1093/heapro/dap032

The traffic light electronic tag. (February 8, 2014). Journal TODAY- News Ecuador. Retrieved from http://www.hoy.com.ec/noticias-ecuador/la-etiqueta-semaforocon-sistema-electronico-600490.html

Verstraeten, R., Van Royen, K., Ochoa-Avilés, A., Penafiel, D., Holdsworth, M., Donoso, S., Maes, L., \& Kolsteren, P. (2014). A conceptual framework for healthy eating behavior in Ecuadorian adolescents: A qualitative study. PLOS ONE, 9 (1), 17. 